\title{
Local Subspace Classifier with Transform-Invariance for Image Classification
}

\author{
Seiji HOTTA $^{\dagger a)}$, Member
}

\begin{abstract}
SUMMARY A family of linear subspace classifiers called local subspace classifier (LSC) outperforms the $k$-nearest neighbor rule $(k N N)$ and conventional subspace classifiers in handwritten digit classification. However, LSC suffers very high sensitivity to image transformations because it uses projection and the Euclidean distances for classification. In this paper, I present a combination of a local subspace classifier (LSC) and a tangent distance (TD) for improving accuracy of handwritten digit recognition. In this classification rule, we can deal with transform-invariance easily because we are able to use tangent vectors for approximation of transformations. However, we cannot use tangent vectors in other type of images such as color images. Hence, kernel LSC (KLSC) is proposed for incorporating transform-invariance into LSC via kernel mapping. The performance of the proposed methods is verified with the experiments on handwritten digit and color image classification.

key words: local subspace classifier, transform-invariance, tangent distance, kernel mapping, image classification
\end{abstract}

\section{Introduction}

Linear subspace method [1]-[4] is a widely used technique in real-life pattern recognition problems because they can achieve dimension reduction and classification concurrently and compute similarity between an input sample and subspaces in parallel. Hence, some extended versions of them such as a local subspace classifier (LSC) [5], [6] were proposed in the past. In LSC, linear manifolds (affine subspaces) of $k$-nearest training samples of an input sample are computed in each class. After that, the input sample is classified into the class to which the nearest manifold belongs. By this classification rule, we can classify high-dimensional data such as images with high recognition accuracy. The paper [5] showed by experiments that LSC outperformed the $k$-nearest neighbor rule $(k \mathrm{NN})$ and conventional subspace classifiers in handwritten digit classification.

In general, transform-invariance is necessary for pattern recognition, especially significant for image classification, so we focused on image classification in the paper. For high accuracy in image classification, we should achieve invariance against transformations such as shift, scaling, rotation, distortion, and thickness [7]. To achieve invariance against such transformations in distance-based classifiers, a tangent distance (TD) [8], [9] was proposed by Simard et al. In TD, image transformations are approximated with a lin-

Manuscript received April 17, 2007.

Manuscript revised October 22, 2007.

${ }^{\dagger}$ The author is with the Institute of Symbiotic Science and Technology, Tokyo University of Agriculture and Technology, Koganei-shi, 184-8588 Japan.

a) E-mail: s-hotta@cc.tuat.ac.jp

DOI: 10.1093/ietisy/e91-d.6.1756 ear tangent plane spanned by tangent vectors of an image. The tangent distance between two images is measured as the minimum distance between their tangent planes. It was verified that TD improved the accuracy of $k \mathrm{NN}$ drastically in handwritten digit classification [8]. However, the recognition rate of $k \mathrm{NN}$ deteriorates when the dimensionality of feature vectors is large [10]. Hence, it can be expected that a combination of LSC and TD can outperform the original LSC and TD.

In this paper, I present a combination of LSC and TD for handwritten digit recognition. The $k$-nearest neighbors of an input sample are selected in each class by using a two-sided tangent distance. Linear manifolds spanned by the selected transformed-neighbor samples are calculated in individual classes. The proposed method classifies the input sample to the class to which the nearest manifold belongs. In this classification rule, we can deal with transforminvariance easily because we are able to use tangent vectors for approximation of transformations. However, we cannot use tangent vectors in other type of images such as color images. Hence, kernel LSC (KLSC) is proposed for incorporating transform-invariance into LSC via kernel mapping. KLSC is derived by the same manner as kernel nonlinear subspace classifiers (KNS) [11], [12]. The performance of the proposed methods is verified with the experiments on handwritten digit and color image classification.

\section{Local Subspace Classifier and Tangent Distance}

Before presenting the proposed method, let us begin with brief reviews of a local subspace classifier (LSC) [5], [6] and a tangent distance (TD) [8], [9].

\section{$2.1 \quad$ LSC}

The LSC technique first searches for the $k$-closest training samples to an input sample in all classes. Next, a $(k-1)$ dimensional linear manifold is spanned by these training samples in each class. Note that the origin point of the linear manifold is the nearest training sample. The classification is then based on the shortest distance from the input sample to these linear manifolds. This process in the class $j$ with $k=3$ is shown in Fig. 1.

For examining the advantages of LSC in image classification, the two-dimensional linear manifold that spanned by three handwritten digit patterns ' 4 ' is shown in Fig. 2. Each of the corners of the triangle represents pure training 


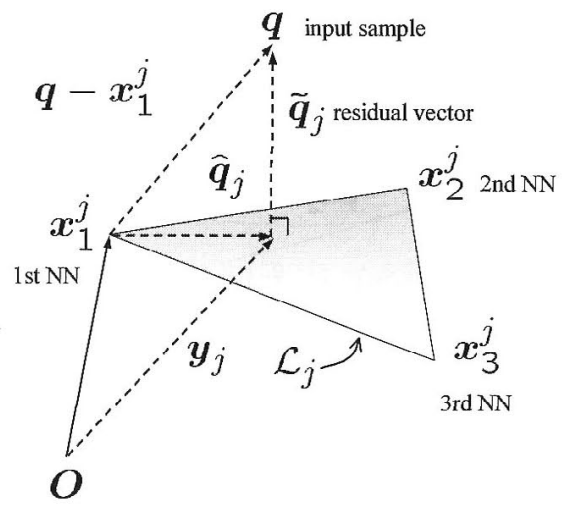

Fig. 1 The concept of LSC in the class $j$ with $k=3$. The input sample $q$ is classified according to the residual length from the input sample $q$ to the linear manifold $\mathcal{L}^{j}$.

samples, whereas the images in between are linear combinations of them. These intermediate images are as good representative samples of the class as the original ones. Hence, an input sample can be considered to have originated from that class when it resembles any of such artificial images [5]. These artificial images interpolate the sparsity between prototypes in high-dimensional space. In addition, LSC uses $k$-neighbor samples, so we can apply LSC to data that is not separable linearly. Based on these properties, LSC can outperform the $k \mathrm{NN}$ rule and conventional subspace classifiers in image classification.

For implementing LSC, I give a procedural formulation of the LSC algorithm. Let $\boldsymbol{x}_{i}^{j}=\left(x_{i 1}^{j} \cdots x_{i d}^{j}\right)^{\top} \in \mathbb{R}^{d}(i=$ $\left.1, \ldots, n^{j}\right)$ be the $i$ th $d$-dimensional training sample belonging to a class $j$, where $n^{j}$ is the number of the training samples belonging to the class $j(j=1, \ldots, C)$.

Step1 When an input sample $\boldsymbol{q}=\left(q_{1} \cdots q_{d}\right)^{\top}$ is given, find then the $k$ training samples closest to the input sample and denote them as $x_{1}^{j}, \ldots, x_{k}^{j}$. Note that the distance between the input sample $\boldsymbol{q}$ and each training sample $\boldsymbol{x}_{i}^{j}$ is measured by the Euclidean distance:

$$
D\left(\boldsymbol{q}, \boldsymbol{x}_{i}^{j}\right)=\left\|\boldsymbol{q}-\boldsymbol{x}_{i}^{j}\right\| \quad\left(i=1, \ldots, n^{j}\right) .
$$

Step2 Form a $d \times(k-1)$ matrix $\left[\left(x_{2}^{j}-x_{1}^{j}\right) \cdots\left(x_{k}^{j}-x_{1}^{j}\right)\right]$ and orthonormalize it by using the Gram-Schmidt process or eigenvalue decomposition. Denote the resulting $d \times(k-1)$ orthonormal basis $\mathbf{U}^{j}=\left[\boldsymbol{u}_{1}^{j} \cdots \boldsymbol{u}_{k-1}^{j}\right]$ of the linear manifold for the class $j$.

Step3 Calculate the residual of the input sample $q$ relative to the linear manifold $\mathcal{L}^{j}$ (see Fig. 1) by the following equation:

$$
\tilde{\boldsymbol{q}}^{j}=\boldsymbol{q}-\boldsymbol{y}^{j}=\boldsymbol{q}-\left(\boldsymbol{x}_{1}^{j}+\hat{\boldsymbol{q}}^{j}\right)=\left(\mathbf{I}-\mathbf{U}^{j}\left(\mathbf{U}^{j}\right)^{\top}\right)\left(\boldsymbol{q}-\boldsymbol{x}_{1}^{j}\right),
$$

where the projection of $\boldsymbol{q}-\boldsymbol{x}_{1}^{j}$ on the manifold is given as

$$
\hat{\boldsymbol{q}}^{j}=\mathbf{U}^{j}\left(\mathbf{U}^{j}\right)^{\top}\left(\boldsymbol{q}-\boldsymbol{x}_{1}^{j}\right) .
$$

Therefore, the input sample $\boldsymbol{q}$ is then classified according to

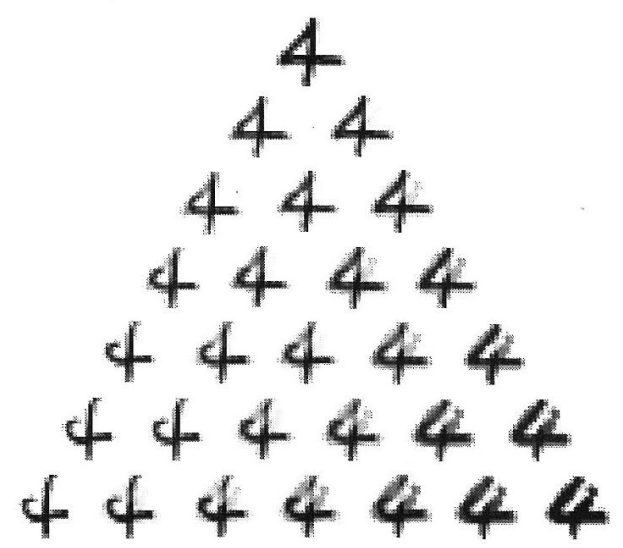

Fig. 2 A piece of the two-dimensional linear manifold spanned by three examples of handwritten digits ' 4 ' in the corners.

the minimal $\left\|\tilde{\boldsymbol{q}}^{j}\right\|$ to the class $j$, i.e., the class of the input sample (denoted $\omega$ ) is given by the following equation:

$$
\begin{aligned}
\omega & =\arg \min _{j=1, \ldots, C}\left\|\tilde{\boldsymbol{q}}^{j}\right\|^{2} \\
& =\arg \min _{j=1, \ldots, C}\left\|\left(\mathbf{I}-\mathbf{U}^{j}\left(\mathbf{U}^{j}\right)^{\top}\right)\left(\boldsymbol{q}-\boldsymbol{x}_{1}^{j}\right)\right\|^{2} \\
& =\arg \min _{j=1, \ldots, C}\left\|\boldsymbol{q}-\boldsymbol{x}_{1}^{j}\right\|^{2}-\left\|\left(\mathbf{U}^{j}\right)^{\top}\left(\boldsymbol{q}-\boldsymbol{x}_{1}^{j}\right)\right\|^{2} .
\end{aligned}
$$

In any case, the residual length from the input sample $q$ to the linear manifold $\mathcal{L}^{j}$ is equal to or smaller than to the nearest training sample, i.e., $\left\|\tilde{\boldsymbol{q}}^{j}\right\| \leq d\left(\boldsymbol{q}, \boldsymbol{x}_{1}^{j}\right)$. When $k=1$, LSC is equivalent to the nearest neighbor rule.

\subsection{TD for Transform-Invariance Digit Classification}

We encounter different types of geometric transformation when dealing with image recognition problems. It is important for us to incorporate transform-invariance into classification rules for achieving high accuracies. Distance-based classification algorithms such as $k \mathrm{NN}$ and RBF classifiers often rely on simple distances such as the Euclidean distance. However, they suffer a high sensitivity to geometric transformation of images such as shifts, scaling and others. To overcome this difficulty, Simard et al. proposed TD for dealing with transform-invariance in distance-based classifiers [8], [9].

For example, when an image $q$ is transformed with small rotations that depend on one parameter $\alpha$, and so the set of all the transformed images is given as a onedimensional curve $S_{q}$ (i.e., a nonlinear manifold) in a pixel space (see from top to middle in Fig. 3). Similarly, assume that the set of all the transformed images of another image $\boldsymbol{x}$ is given as a one-dimensional curve $S_{x}$. In this situation, we can regard the distance between manifolds $S_{q}$ and $S_{x}$ as an adequate dissimilarity for two images $\boldsymbol{q}$ and $\boldsymbol{x}$, respectively. For computational reasons, we measure the distance between the corresponding tangent planes instead of calculating the distance between their nonlinear manifolds (see Fig. 4). The manifold $S_{q}$ is approximated by its tangent hyperplane at point $\boldsymbol{q}$ : 


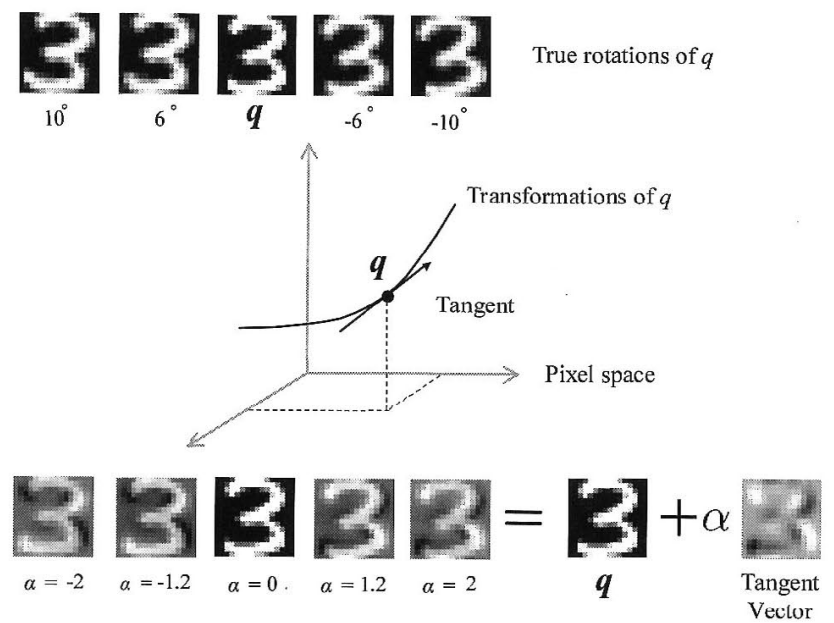

Fig. 3 Top: Small rotations of an original image. Middle: Representation of the effect of the rotation in pixel space. Bottom: Images obtained by adding various amounts $(\alpha)$ of the tangent vector.

$$
S_{q} \simeq \boldsymbol{q}+\sum_{i=1}^{r} \alpha_{i}^{q} \boldsymbol{t}_{i}^{q}=\boldsymbol{q}+\mathbf{T}_{q} \alpha_{q}
$$

where $t_{i}^{q}$ is the $i$ th $d$-dimensional tangent vector (TV) that spans the $r$-dimensional tangent hyperplane (i.e., the number of transformation is $r$ ) at point $\boldsymbol{q}$ and the $\alpha_{i}^{q}$ is its corresponding parameter. The notations $\mathbf{T}_{q}$ and $\boldsymbol{\alpha}_{q}$ denote $\mathbf{T}_{q}=\left(\boldsymbol{t}_{1}^{q} \cdots \boldsymbol{t}_{r}^{q}\right)$ and $\boldsymbol{\alpha}_{q}=\left(\alpha_{1}^{q} \cdots \alpha_{r}^{q}\right)$, respectively.

For approximating $S_{q}$, we compute TVs in advance by using finite difference. For instance, the seven TVs for the image depicted in Fig. 3 are shown in Fig. 5. These TVs are derived from the Lie group theory (thickness deformation is an exceptional case), so the maximum number of transformations $r$ is seven (see [9] for details). Of course, the $r$ has the effect on classification performance, but the influence of $r$ on accuracy has never been shown before, so it is investigated in experiments of this paper. Small transformation of $q$ can be obtained by a linear combination of the original image $\boldsymbol{q}$ and these TVs. For example, the linear combinations with different amounts of $\alpha$ of the TV for rotation are shown in the bottom in Fig. 3.

For dealing with invariance in distance-based classifiers, we compute distance called two sided TD:

$$
d_{2 S}^{2}(\boldsymbol{q}, \boldsymbol{x})=\min _{\boldsymbol{\alpha}_{q}, \boldsymbol{\alpha}_{x}}\left\|\boldsymbol{q}+\mathbf{T}_{q} \boldsymbol{\alpha}_{q}-\left(\boldsymbol{x}+\mathbf{T}_{x} \boldsymbol{\alpha}_{x}\right)\right\|^{2} .
$$

The above minimization is simple since the squared distance is a quadratic function of $\boldsymbol{\alpha}$. The solutions of $\boldsymbol{\alpha}_{q}$ and $\alpha_{x}$ can be derived as follows:

$$
\begin{aligned}
& \boldsymbol{\alpha}_{q}=\left(\mathbf{T}_{q x} \mathbf{T}_{x x}^{-1} \mathbf{T}_{x q}-\mathbf{T}_{q q}\right)^{-1}\left(\mathbf{T}_{q x} \mathbf{T}_{x x}^{-1} \mathbf{T}_{x}^{\top}-\mathbf{T}_{q}^{\top}\right)(\boldsymbol{x}-\boldsymbol{q}), \\
& \boldsymbol{\alpha}_{x}=\left(\mathbf{T}_{x x}-\mathbf{T}_{x q} \mathbf{T}_{q q}^{-1} \mathbf{T}_{q x}\right)^{-1}\left(\mathbf{T}_{x q} \mathbf{T}_{q q}^{-1} \mathbf{T}_{q}^{\top}-\mathbf{T}_{x}^{\top}\right)(\boldsymbol{x}-\boldsymbol{q}),
\end{aligned}
$$

where $\mathbf{T}_{q q}=\mathbf{T}_{q}^{\top} \mathbf{T}_{q}, \mathbf{T}_{x q}=\mathbf{T}_{x}^{\top} \mathbf{T}_{q}, \mathbf{T}_{q x}=\mathbf{T}_{q}^{\top} \mathbf{T}_{x}, \mathbf{T}_{x x}=\mathbf{T}_{x}^{\top} \mathbf{T}_{x}$.

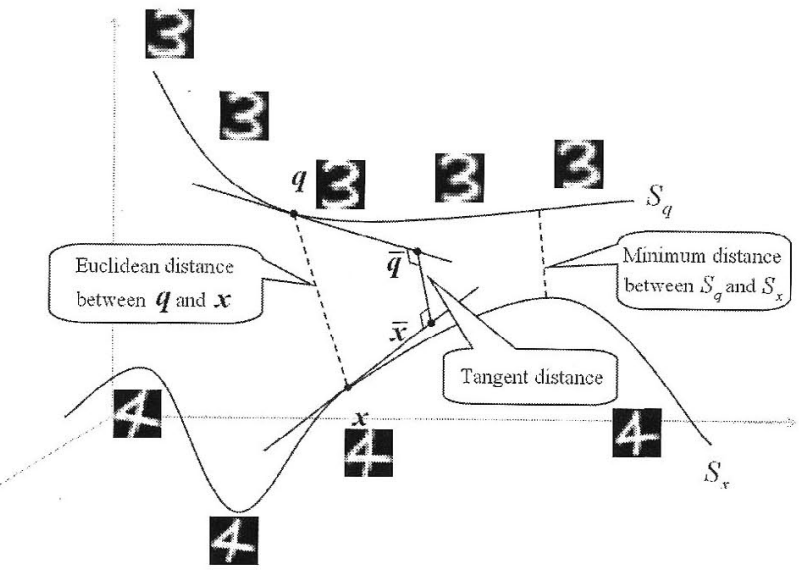

Fig. 4 Illustration of the Euclidean distance and the tangent distance between $\boldsymbol{q}$ and $\boldsymbol{x}$. Black dots denote the transformed-patterns on tangent hyperplanes that minimize two sided TD.

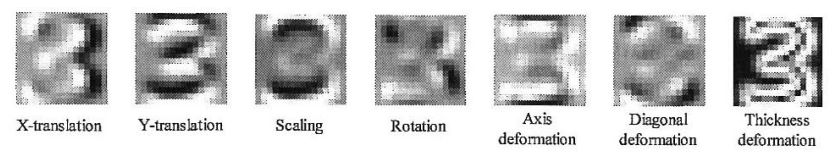

Fig. 5 Tangent vectors $\boldsymbol{t}_{i}$ for the pattern in Fig. 3. From left to right, they denote $x$-translation, $y$-translation, scaling, rotation, axis deformation, diagonal deformation and thickness deformation, respectively.

The experiments on handwritten digit recognition in [8] showed that $k \mathrm{NN}$ with TD achieves higher accuracy than the use of the Euclidean distance.

\section{LSC with Transform-Invariance for Image Classifi- cation}

Here, I propose two modified LSC approaches for incorporating transform-invariance into LSC for image classification. The combination of a LSC and TD is first proposed for handwritten digit recognition. By this proposal, we can achieve transform-invariance in digit recognition because we can use tangent vectors for approximation of transformations. However, we cannot use tangent vectors in other type of images such as color images, so I propose kcrncl LSC (KLSC) to incorporate transform-invariance via kernel mapping. The KLSC method is a very general classifier, but we must select an appropriate kernel function for high accuracy. Hence, I introduce a tangent distance kernel (TDK) [13] and gencralized histogram intersection (GHI) kernel [14] for handwritten digit and color image classification, respectively.

\subsection{LSC with TD}

I first propose a combination of linear LSC and TD for handwritten digit pattern classification. In general, we should not compute the distance between two patterns until we had transformed them to be as similar to one another as possible [7]. However, we uncritically measure the Euclidean distance between samples in LSC, so there is a possibility that 
LSC classifies an input sample by using the training samples that are not similar to the input sample. On the other hand, Simard et al. investigated the performance of TD using $k \mathrm{NN}$ but the recognition rate of $k \mathrm{NN}$ deteriorates when the dimensionality of feature vectors is large [10]. Hence, I combine LSC and TD for overcoming difficulties found in LSC and TD. In short, proposed classification is shown as follows:

Step1 Find $k$-nearest training samples to an input sample in the class $j$ according to $d_{2 S}^{2}$ instead of the Euclidean distance. Store the $k$ transformed-images on tangent hyperplanes (cf. black dots in Fig. 4):

$$
\begin{aligned}
& \overline{\boldsymbol{q}}_{i}^{j}=\boldsymbol{q}+\mathbf{T}_{q} \boldsymbol{\alpha}_{i}^{j}(i=1, \ldots, k), \\
& \overline{\boldsymbol{x}}_{i}^{j}=\boldsymbol{x}_{i}^{j}+\mathbf{T}_{x_{i}}^{j} \boldsymbol{\alpha}_{x_{i}}^{j}(i=1, \ldots, k),
\end{aligned}
$$

where $\boldsymbol{\alpha}_{i}^{j}$ is the coefficient vector for $\boldsymbol{q}$ that minimizes $d_{2 S}^{2}$ between $\boldsymbol{q}$ and $\boldsymbol{x}_{i}^{j}$ (cf. Eq. 7). The notations $\mathbf{T}_{x_{i}}^{j}$ and $\boldsymbol{\alpha}_{x_{i}}^{j}$ denote TV and its corresponding coefficient vector for $\boldsymbol{x}_{i}^{j}$ that minimizes $d_{2 S}^{2}$ between $\boldsymbol{q}$ and $\boldsymbol{x}_{i}^{j}$ (cf. Eq. 8), respectively.

Step 2 Form a $d \times(k-1)$ matrix $\left[\left(\bar{x}_{2}^{j}-\bar{x}_{1}^{j}\right) \cdots\left(\bar{x}_{k}^{j}-\bar{x}_{1}^{j}\right)\right]$. Then orthonormalize it and denote the resulting orthonormal basis of the linear manifold for the class $j$ by $\mathbf{U}^{j}=$ $\left[\boldsymbol{u}_{1}^{j} \cdots \boldsymbol{u}_{k-1}^{j}\right]$.

Step3 Compute the mean sample of $k$ transformedinput-samples $\overline{\boldsymbol{q}}_{1}^{j}, \ldots, \overline{\boldsymbol{q}}_{k}^{j}$ and use it as the new input sample for the class $j$ because an input sample should be represented as one point in pixel space for the LSC classifier. The mean input sample is given by the following equation:

$$
\boldsymbol{m}^{j}=\frac{1}{k} \sum_{i=1}^{k} \overline{\boldsymbol{q}}_{i}^{j}=\boldsymbol{q}+\mathbf{T}_{q} \frac{1}{k} \sum_{i=1}^{k} \boldsymbol{\alpha}_{i}^{j}
$$

Step 4 Calculate the residual length from the new input sample $\boldsymbol{m}^{j}$ to the linear manifold $\mathcal{L}^{j}$ and classify the input sample to the class that minimizes the residual length, i.e., the class of the input sample $\omega$ is give by the following equation:

$$
\omega=\arg \min _{j=1, \ldots, C}\left\|\boldsymbol{m}^{j}-\bar{x}_{1}^{j}\right\|^{2}-\left\|\left(\mathbf{U}^{j}\right)^{\top}\left(\boldsymbol{m}^{j}-\overline{\boldsymbol{x}}_{1}^{j}\right)\right\|^{2} .
$$

Note that it is difficult to incorporate transform-invariance into conventional subspace classifiers such as CLAFIC [1] because such classifiers do not directly measure distance between an input sample and training ones.

\subsection{Kernel LSC (KLSC)}

For incorporating transform-invariance into LSC via kernel mapping [15], I derive a kernel LSC (KLSC). The extension from a linear classifier to nonlinear one can be achieved by a kernel trick $\Phi(\boldsymbol{x})^{\top} \Phi(\boldsymbol{y})=K(\boldsymbol{x}, \boldsymbol{y})$ for mapping samples from an input space to a feature space $\mathbb{R}^{d} \mapsto \mathcal{F}$. In addition, we make use of the results of kernel PCA [16] for computing the residual length from an input sample to linear manifolds in $\mathcal{F}$. In brief, the algorithm of KLSC is given as follows:
Step1 Find the $k$ training samples closest to an input sample $\Phi(\boldsymbol{q})$ in $\mathcal{F}$ and denote them as $\Phi\left(\boldsymbol{x}_{1}^{j}\right), \ldots, \Phi\left(\boldsymbol{x}_{k}^{j}\right)$. Note that the Euclidean distance between the input sample $\Phi(\boldsymbol{q})$ and the training sample $\Phi\left(\boldsymbol{x}_{i}^{j}\right)$ in $\mathcal{F}$ is given by the following equation:

$$
\begin{aligned}
& D^{2}\left(\Phi(\boldsymbol{q}), \Phi\left(\boldsymbol{x}_{i}^{j}\right)\right)=\left\|\Phi(\boldsymbol{q})-\Phi\left(\boldsymbol{x}_{i}^{j}\right)\right\|^{2} \\
& =K(\boldsymbol{q}, \boldsymbol{q})-2 K\left(\boldsymbol{q}, \boldsymbol{x}_{i}^{j}\right)+K\left(\boldsymbol{x}_{i}^{j}, \boldsymbol{x}_{i}^{j}\right) .
\end{aligned}
$$

Step 2 Let $\mathbf{X}^{j}$ be the basis of vectors in $\mathcal{F}$, i.e., $\mathbf{X}^{j}=$ $\left[\left(\Phi\left(\boldsymbol{x}_{2}^{j}\right)-\Phi\left(\boldsymbol{x}_{1}^{j}\right)\right) \cdots\left(\Phi\left(\boldsymbol{x}_{k}^{j}\right)-\Phi\left(\boldsymbol{x}_{1}^{j}\right)\right)\right]$. Form a $(k-1) \times(k-1)$ matrix $\mathbf{N}^{j}=\left(\mathbf{X}^{j}\right)^{\top} \mathbf{X}^{j}$, where the $(m, n)$ element of $\mathbf{N}^{j}$ is given as follows:

$$
\begin{aligned}
N_{m n}^{j} & =K\left(\boldsymbol{x}_{m+1}^{j}, \boldsymbol{x}_{n+1}^{j}\right)-K\left(\boldsymbol{x}_{m+1}^{j}, \boldsymbol{x}_{1}^{j}\right) \\
& -K\left(\boldsymbol{x}_{1}^{j}, \boldsymbol{x}_{n+1}^{j}\right)+K\left(\boldsymbol{x}_{1}^{j}, \boldsymbol{x}_{1}^{j}\right) .
\end{aligned}
$$

Decompose $\mathbf{N}^{j}$ by using eigenvalue decomposition and form a $(k-1) \times(k-1)$ orthonormal matrix $\mathbf{V}^{j}$ using the eigenvectors of $\mathbf{N}^{j}\left(v_{1}^{j}, \ldots, v_{k-1}^{j}\right)$ normalized with the corresponding eigenvalues $\left(\lambda_{1}^{j}, \ldots, \lambda_{k-1}^{j}\right)$ such as

$$
\mathbf{V}^{j}=\left[\boldsymbol{v}_{1}^{j} / \sqrt{\lambda_{1}^{j}} \cdots v_{k-1}^{j} / \sqrt{\lambda_{k-1}^{j}}\right]
$$

This orthonormal matrix represents the orthonormal basis of the linear manifold for the class $j$ in feature space $\mathcal{F}$ as $\mathbf{U}^{j}=\mathbf{X}^{j} \mathbf{V}^{j}$. Note that we cannot represent $\mathbf{U}^{j}$ as a matrix explicitly due to its high-dimensionality.

Step 3 The input sample $\Phi(\boldsymbol{q})$ is classified according to the minimal residual length to the linear manifolds in $\mathcal{F}$, i.e., the class of the input sample $\omega$ is given by the following equation:

$$
\begin{aligned}
\omega= & \arg \min _{j=1, \ldots, C}\left\{\left\|\Phi(\boldsymbol{q})-\Phi\left(\boldsymbol{x}_{1}^{j}\right)\right\|^{2}\right. \\
& \left.-\left\|\left(\mathbf{U}^{j}\right)^{\top}\left(\Phi(\boldsymbol{q})-\Phi\left(\boldsymbol{x}_{1}^{j}\right)\right)\right\|^{2}\right\} \\
= & \arg \min _{j=1, \ldots, C}\left\{D^{2}\left(\Phi(\boldsymbol{q}), \Phi\left(\boldsymbol{x}_{1}^{j}\right)\right)-\left\|\left(\mathbf{V}^{j}\right)^{\top} \boldsymbol{Q}^{j}\right\|^{2}\right\},
\end{aligned}
$$

where $Q^{j}$ is a $(k-1) \times 1$ vector of which the $i$ th element is given by following equation:

$$
\begin{aligned}
& Q_{i}^{j}=\left(\Phi\left(\boldsymbol{x}_{i+1}^{j}\right)-\Phi\left(\boldsymbol{x}_{1}^{j}\right)\right)^{\top}\left(\Phi(\boldsymbol{q})-\Phi\left(\boldsymbol{x}_{1}^{j}\right)\right) \\
& =K\left(\boldsymbol{x}_{i+1}^{j}, \boldsymbol{q}\right)-K\left(\boldsymbol{x}_{i+1}^{j}, \boldsymbol{x}_{1}^{j}\right)-K\left(\boldsymbol{x}_{1}^{j}, \boldsymbol{q}\right)+K\left(\boldsymbol{x}_{1}^{j}, \boldsymbol{x}_{1}^{j}\right) .
\end{aligned}
$$

If necessary, we reduce the dimensionality of a linear manifold on the basis of a criterion such as a cumulative proportion. Note that the accuracy of KLSC is sensitive against not the dimensionality of a linear manifold but the number of $k$-nearest neighbors. The KLSC technique uses few training samples (i.e. $k$-nearest samples, $k \ll n^{j}$ ) for computing a linear manifold, and so the rank of the linear manifold is equal to or less than $k-1$. In contrast, if $k$ is equal to $n^{j}$ in all classes, the result on KLSC then is equivalent to that of the kernel projection distance method proposed in [12].

For incorporating transform-invariance into kernel classifiers for digit classification, some kernels have been 
proposed in the past [13], [17]. Here, we focus on a tangent distance kernel (TDK) proposed by Haasdonk et al. [13] because of the reasonable performance that can be obtained in spite of their extreme simplicity. TDK is defined by replacing the Euclidean distance with a TD-measure in arbitrary distance-based kernels. For example, if we modify the following radial basis function (RBF) kernel

$$
K(\boldsymbol{x}, \boldsymbol{y})=\exp \left(-\beta\|\boldsymbol{x}-\boldsymbol{y}\|^{2}\right)
$$

by replacing the Euclidean distance with a two sided TD, we then obtain the kernel called two sided TD kernel:

$$
K(\boldsymbol{x}, \boldsymbol{y})=\exp \left(-\beta \times d_{2 S}^{2}(\boldsymbol{x}, \boldsymbol{y})\right) .
$$

We can achieve higher accuracy by this simple modification than the use of the original RBF kernel [13]. In addition, the above modification is adequate for kernel setting because of its natural definition and symmetric property. Hence, I used the two sided TDK on experiments of this paper.

For other type of images such as color images, we must select an appropriate kernel function for high accuracy. In this paper, we focus on color image classification as an example. For color image classification, color information is as important as shape information and can be easily extracted from images. Hence, we frequently use color histograms for representation of color images. In fact, color histograms give good results. For example, it is well known that the similarity measure called histogram intersection (HI) [18] is effective in color image retrieval because HI is robust to geometric transformations such as distractions, view point changing, and occlusion. Hence, we incorporate $\mathrm{HI}$ measure into KLSC by using a generalized histogram intersection (GHI) kernel [14]. The GHI kernel is given by the following equation:

$$
K(\boldsymbol{x}, \boldsymbol{y})=\sum_{i=1}^{d} \min \left(\left|x_{i}\right|^{\gamma},\left|y_{i}\right|^{\gamma}\right),
$$

where $\boldsymbol{x}=\left(x_{1} \cdots x_{m}\right)$ and $\boldsymbol{y}=\left(y_{1} \cdots y_{m}\right)$ are two color histograms with $m$-bins. In addition, it is known that the accuracy of GHI was higher than that of $\mathrm{HI}$ because power mapping (i.e., hyper-parameter $\gamma>0$ ) helps us to separate data in a mapping space. The paper [14] reported that the power mapping is effective in improvement in recognition performance.

\section{Experiments}

I show the experimental results on handwritten digit and color image classification. In experiments, the dimensionality of a linear manifold in LSC and KLSC was always set as $k-1$. All methods were implemented with MATLAB on a standard PC that has Pentium $3 \mathrm{GHz}$ CPU and 1 GB RAM.

\subsection{Handwritten Digit Classification}

I first tested proposed classifiers on MNIST [19]. The

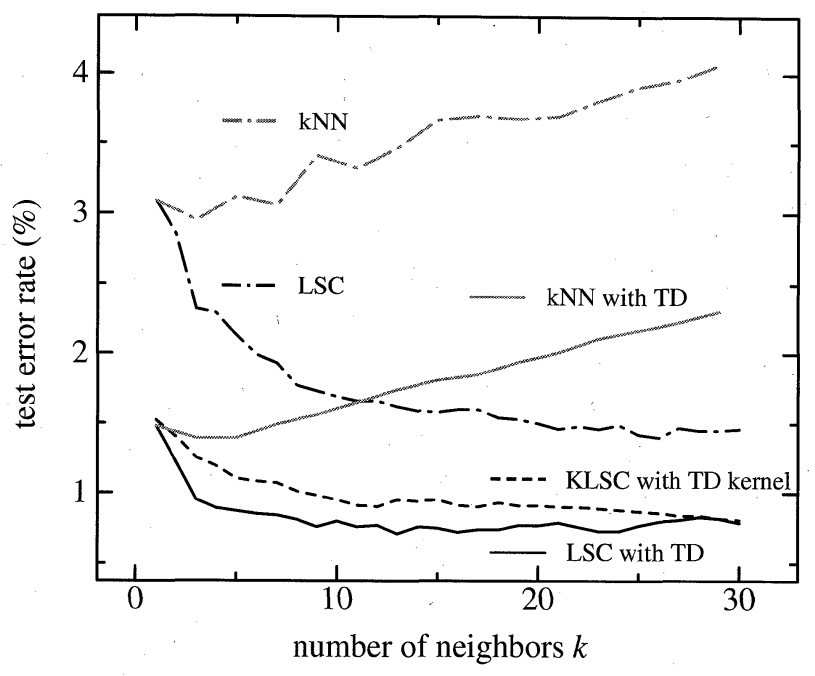

Fig. 6 Relationship between $k$ and error rates on MNIST.

MNIST dataset consists of 60,000 training and 10,000 test images. In this experiment, we reversed the intensity of $28 \times 28$ images to represent the background of them with black.

Figure 6 shows the relationship between the number of neighbors $k$ and error rates of each classifier: LSC, combination of LSC and TD (LSC with TD), KLSC with TDK, $k \mathrm{NN}$, and $k \mathrm{NN}$ with TD. As shown in the figure, the error rates of the LSC-based classifiers against test samples decreased as the $k$ increased. Note that the test error rates of the LSC-based classifiers were not sensitive against $k$ as compared with those of the $k \mathrm{NN}$-based classifiers. In addition, the error rate of LSC with TD was lower than those of LSC and KLSC in every $k$. Hence, it can be considered that selection of $k$ on LSC with TD is easier than selection of $k$ on the others.

Table 1 lists the lowest error rates with and without transform-invariance on each classifier: $k \mathrm{NN}$, projection distance method (PDM) [3], LSC, SVM, kernel PDM (KPDM) [12] and KLSC. The parameter $\sigma$ indicate the variance of the Gauss filter for computing tangent vectors. The number of neighbors $k$ in $k \mathrm{NN}$ and the LSC-based classifiers were tuned on a separate validation set (50000 training samples, 10000 validation samples). For computational reason, the dimensionality of subspaces in KPDM was determined by a cumulative proportion (denoted as c.p.). In contrast, we selected the dimensionality of subspaces in PDM by hand. The results on SVM is referred to [17]. Due to out of memory, the result on KPDM was not obtained. Table 1 shows that all error rates improved by using transform-invariance. Furthermore, LSC with TD outperformed the separate use of LSC and TD. In comparison with other methods, LSC with TD achieved lowest error rates. Note that the error rate of LSC with TD was lower than that of KLSC because classspecific deformation was added to not only training samples but also an input one. This result indicates that we can achieve high accuracy without kernel mapping if we design 
Table 1 Test error rates on MNIST.

\begin{tabular}{|c|c|c|c|c|}
\hline method & without transform-invariance & error [\%] & with transform-invariance & error [\%] \\
\hline$k$ NN & Euclidean Distance $(k=3)$ & 3.0 & Tangent Distance $(k=3, \sigma=0.9)$ & 1.4 \\
\hline PDM & dimensionality for subspaces is 26 & 4.1 & - & - \\
\hline LSC & Euclidean Distance $(k=26)$ & 1.4 & Tangent Distance $(k=13, \sigma=0.7)$ this work & $\mathbf{0 . 7}$ \\
\hline SVM & Polynomial kernel $[17]$ & 1.4 & Virtual polynomial SVM [17] & 0.8 \\
\hline KPDM & out of memory & - & out of memory & - \\
\hline KLSC & RBF kernel $\left(\beta=10^{-7}, k=18\right)$ this work & 1.4 & TD kernel $\left(K_{2 S}^{\mathrm{RBF}}, k=30, \beta=10^{-7}\right)$ this work & 0.8 \\
\hline
\end{tabular}

Table 2 Test error rates on USPS.

\begin{tabular}{|c|c|c|c|c|}
\hline method & without transform-invariance & error [\%] & with transform-invariance & error [\%] \\
\hline$k$ NN & Euclidean Distance $(k=1)$ & 5.5 & Tangent Distance $(k=1, \sigma=0.7)$ & 2.9 \\
\hline PDM & dimensionality for subspaces is 30 & 5.2 & - & - \\
\hline LSC & Euclidean Distance $(k=11)$ & 4.0 & Tangent Distance $(k=7, \sigma=1.1)$ this work & $\mathbf{2 . 1}$ \\
\hline SVM & RBF kernel $[13]$ & 4.6 & TD kernel $[13]$ & 3.8 \\
\hline KPDM & RBF kernel (c.p. $\left.=0.9, \beta=10^{-6}\right)$ & 4.4 & TD kernel $\left(\right.$ c.p. $\left.=0.6, \beta=10^{-6}\right)$ & 3.3 \\
\hline KLSC & RBF kernel $\left(k=10, \beta=10^{-9}\right)$ this work & 3.9 & TD kernel $\left(k=5, \beta=10^{-6}\right)$ this work & 2.6 \\
\hline
\end{tabular}

a classifier with the appropriate use of transform-invariance.

Next, I tested proposed classifiers on the USPS dataset [20]. The number of training samples of USPS is fewer than that of MNIST, so this dataset is more difficult to recognize than MNIST. The USPS consists of 7,291 training and 2,007 test images. Table 2 lists the lowest error rates with the parameter values of each classifier. The numbers of neighbors $k$ in $k \mathrm{NN}$ and the LSC-based classifiers were tuned on a separate validation set (6291 training samples, 1000 validation samples). The results of TD is different from the paper [8] because the experiment in [8] was performed using an extended training set. The results on SVM is referred to [13]. Table 2 shows that proposed LSC with TD outperformed the other classifiers. Furthermore, the result on LSC with TD was almost the same as the lowest error rate on USPS (1.9\%) reported in [21].

Finally, I investigated the influence of the number of tangent vectors $r$ in LSC with TD using the USPS dataset. Figure 7 shows test error rates with respect to the number of tangent vectors $r$ in LSC with TD. The vertical and horizontal axes represent test error rates and the number of tangent vectors $r$, respectively. The test error rates with $r=1$ and $r=7$ are equivalent to the results of LSC and LSC with $\mathrm{TD}$, respectively. The other error rates were calculated by averaging error rates obtained by changing combinations of tangent vectors. I used $\sigma=0.7$ and selected $k$ that obtained the best averaged error rates in each $r$. As shown in this figure, test error rate decreased as $r$ increased. This result concluded that we should use all the tangent vectors derived from the Lie group theory for high recognition performance.

\subsection{Color Image Classification}

I applied KLSC to the WANG color image dataset [22] for evaluation of classification performance. The dataset is formed by 10 image classes, African people, beach, buildings, buses, dinosaurs, elephants, flowers, horses, mountains, food, as shown in Fig 8. Each class contains $100 \mathrm{im}$ ages. Images are described using RGB color histogram with a size of $4^{3}=64$ bins. I applied a leave-one-out method for

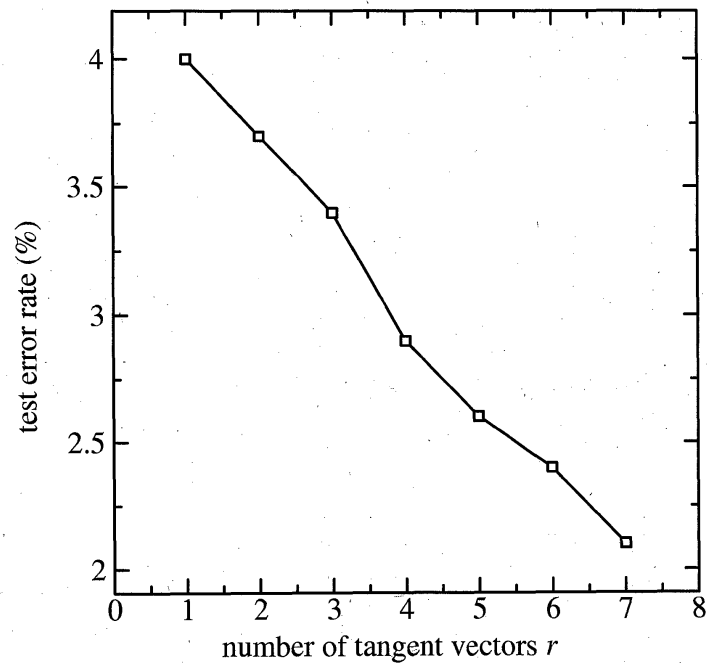

Fig. 7 Test error rate with respect to the number of tangent vectors $r$ in LSC with TD.

estimating error rates.

Table 3 summarizes the best error rates on each classifier. In SVM, the parameter $C$ indicates the soft margin constant. For SVM, I used the SVM package called LIBSVM [23]. In linear LSC, replacing the Euclidean distance with histogram intersection was ineffective in improving classification accuracy. However, higher accuracy was achieved in KLSC with GHI kernel than the case of using RBF kernel i.e., the use of transform-invariance via appropriate kernel mapping helped improve recognition performance. In other words, we must select an appropriate kernel function for high accuracy with KLSC. In addition, KLSC outperformed other classifiers such as SVM, i.e., this good performance is due to the superior generalization ability of LSC.

\section{Conclusions}

This paper presented a combination of a local subspace classifier (LSC) and a tangent distance (TD) for handwritten 
Table 3 Error rates on WANG dataset [22].

\begin{tabular}{|c|c|c|c|c|}
\hline method & without transform-invariance & error [\%] & with transform-invariance & error [\%] \\
\hline$k$ NN & Euclidean Distance $(k=1)$ & 31.6 & Histogram Intersection $(k=1)$ & 25.1 \\
\hline PDM & dimensionality for subspaces is 2 & 30.5 & & - \\
\hline LSC & Euclidean Distance $(k=5)$ & 26.2 & Histogram Intersection $(k=7)$ & 26.8 \\
\hline SVM & RBF kernel $(C=100, \beta=2)$ & 19.2 & GHI kernel $(C=0.25, \gamma=0.28)$ & 12.4 \\
\hline KPDM & RBF kernel $($ c.p. $=0.95, \beta=1)$ & 21.2 & GHI kernel $($ c.p. $=0.95, \gamma=0.33)$ & 12.7 \\
\hline KLSC & RBF kernel $(k=55, \beta=0.5)$ this work & 20.5 & GHI kernel $(k=16, \gamma=0.35)$ this work & $\mathbf{1 1 . 3}$ \\
\hline
\end{tabular}

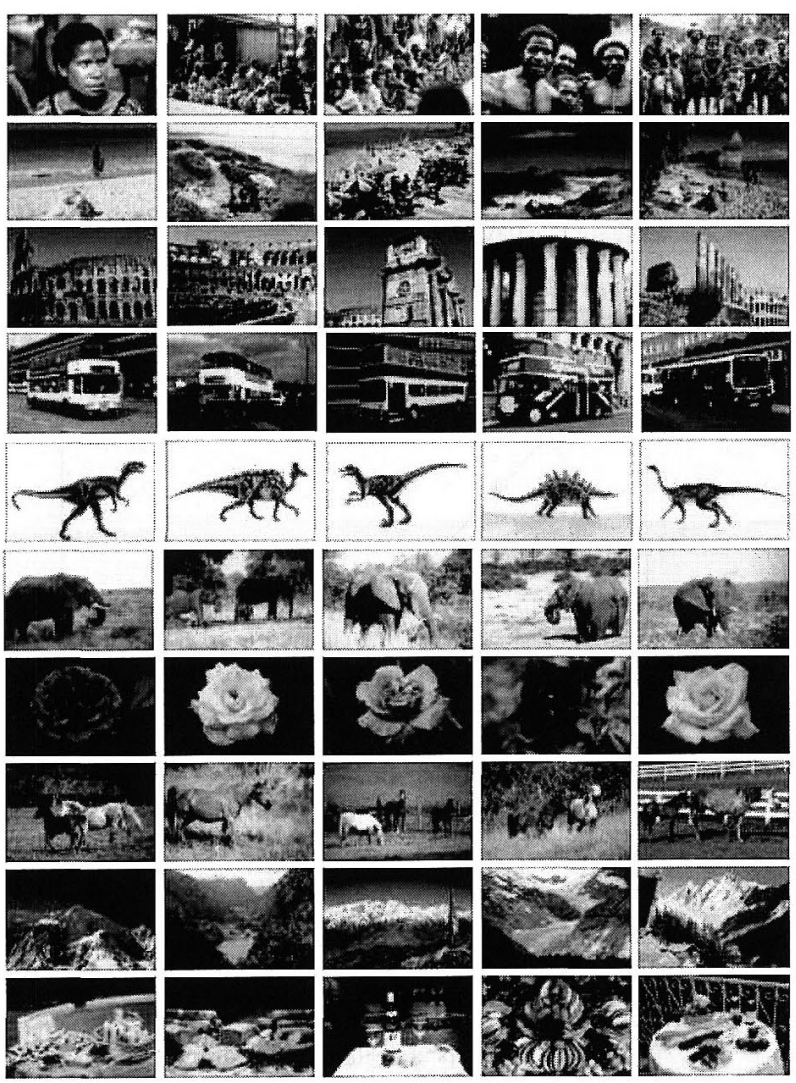

Fig. 8 Each row presents one of the 10 classes used for experiments (African people, beach, buildings, buses, dinosaurs, elephants, flowers, horses, mountains, food).

digit recognition. In addition, I derived kernel LSC to incorporate transform-invariance into LSC via kernel mapping. In experiments, LSC with TD achieved the best performance for handwritten digit classification, and for the color image classification the best performance was obtained by KLSC with a generalized histogram intersection kernel. These results indicate that we can achieve high accuracy without kernel mapping if we can incorporate transform-invariance into a classifier directly. However, if we cannot do it, then kernel mapping is useful for improving accuracy. Hence, we should select a suitable approach based on the situation of classification problems.

The advantageous points on LSC are that LSC requires small amount of memory and is not a binary classifier. The amount of memory requirement on LSC is $d \times k$ per one class, where $d$ and $k$ are the dimensionality of a pattern and the number of neighbors, respectively. When $k \ll N$ ( $N$ is the total number of training samples) is satisfied, the memory cost of LSC then gets lower than that of a kernel subspace classifier $(N \times N)$. Furthermore, LSC is suitable for multi-class classification problems because it is not a binary classifier.

On the other hand, the classification cost of LSC is high because subspaces are designed after an input sample is observed. However, we can calculate manifolds on individual classes independently, so it is possible to reduce calculation time by using parallel processing. Future work will be dedicated to speed up the test phase and improve the proposed classifiers for pattern classification other than images.

\section{References}

[1] S. Watanabe, P.F. Lambert, C.A. Kulikowski, J.L. Buxton, and R. Walker, "Evaluation and selection of variables in pattern recognition," Computer and Information Sciences, vol.2, pp.91-122, 1967.

[2] T. Iijima, H. Genchi, and K. Mori, "A theory of character recognition by pattern matching method," Proc. 1st Int'1 I. Conf. on Pattern Recognition, pp.50-56, 1973.

[3] K. Ikeda, H. Tanaka, and T. Motooka, "Projection distance method for recognition of hand-written characters," J. IPSJ, vol.24, no.1, pp.106-112, 1983.

[4] E. Oja, Subspace methods of pattern recognition, Research Studies Press, 1983.

[5] J. Laaksonen, Subspace classifiers in recognition of handwritten digits, $\mathrm{PhD}$ thesis, Helsinki University of Technology, 1997.

[6] J. Laaksonen, “Local subspace classifier," Proc. ICANN'97, pp.637-642, 1997.

[7] R.O. Duda, P.E. Hart, and D.G. Stork, Pattern classification, 2nd ed., John Wiley \& Sons, 2001.

[8] P.Y. Simard, Y. LeCun, and J.S. Denker, "Efficient pattern recognition using a new transformation distance," Proc. NIPS, vol.5, pp.50$58,1993$.

[9] P.Y. Simard, Y. LeCun, J.S. Denker, and B. Victorri, “Transformation invariance in pattern recognition - Tangent distance and tangent propagation," Int. J. Imaging Systems and Technology, vol.11, no.3, pp.184-197, 2001.

[10] K. Fukunaga, "Bias of nearest neighbour error estimation," IEEE Trans. Pattern Anal. Mach. Intell., vol.9, no.1, pp.103-112, 1987.

[11] K. Tsuda, "Subspace method in the Hilbert space," IEICE Trans. Inf. \& Syst. (Japanese Edition), vol.J82-D-II, no.4, pp.592-599, April 1999.

[12] E. Maeda and H. Murase, "Kernel based nonlinear subspace method for pattern recognition," IEICE Trans. Inf. \& Syst. (Japanese Edition), vol.J82-D-II, no.4, pp.600-612, April 1999.

[13] B. Haasdonk and D. Keysers, "Tangent distance kernels for support vector machines," Proc. ICPR, vol.2, pp.864-868, 2002.

[14] S. Boughorbel, J.-P. Tarel, and N. Boujemaa, "Generalized histogram intersection kernel for image recognition," Proc. ICIP, vol.3, pp.161-164, 2005.

[15] B. Schölkopf and A.J. Smola, Learning with kernels, MIT Press, 2002. 
[16] B. Schölkopf, A.J. Smola, and K.-R. Müller, "Nonlinear component analysis as a kernel eigenvalue problem," Neural Comput., vol.10, pp.1299-1319, 1998.

[17] B. Schölkopf, P. Simard, A. Smola, and V. Vapnik, "Prior knowledge in support vector kernels," Proc. NIPS, vol.10, pp.640-646, 1998.

[18] M. Swain and D. Ballard, "Color indexing," Int. J. Comput. Vis., vol.7, pp.11-132, 1991.

[19] Y. LeCun, L. Bottou, Y. Bengio, and P. Haffner, "Gradient-based learning applied to document recognition," Intell. Signal Process., pp.306-351, 2001.

[20] Y. LeCun, B. Boser, J.S. Denker, D. Henderson, R.E. Howard, W. Hubbard, and L.D. Jackel, "Backpropagation applied to handwritten zip code recognition," Neural Comput., vol.1, no.4, pp.541-551, 1989.

[21] D. Keysers, C. Gollan, and H. Ney, "Local context in non-linear deformation models for handwritten character recognition," Proc. ICPR, vol.4, pp.511-514, 2004.

[22] J.Z. Wang, J. Li, and G. Wiederhold, "SIMPLIcity: Semanticssensitive integrated matching for picture libraries approach," IEEE Trans. Pattern Anal. Mach. Intell., vol.23, no.9, pp.947-963, 2001.

[23] C.C. Chang and C.J. Lin, "LIBSVM: A library for support vector machines," 2001. Software available at http://www.csie.ntu.edu.tw/ cjlin/libsvm

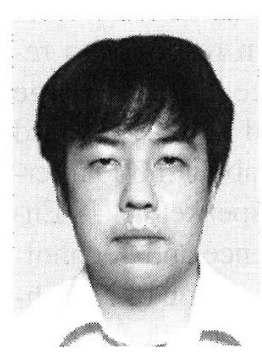

Seiji Hotta received B.E., M.E., and Dr.Eng. degrees from Kyushu Institute of Design in 1998, 1999 and 2002, respectively. He is currently an associate professor at Institute of Symbiotic Science and Technology, Tokyo University of Agriculture and Technology. His research interests include pattern recognition, image and video retrieval. Dr. Hotta is a member of IEEE, ITE, IPSJ. 